\title{
Uso da cognitive orientation to daily occupational performance (co-op) com crianças com transtorno do desenvolvimento da coordenação*
}

\author{
Cognitive orientation to daily occupational \\ performance (co-op) with children with \\ developmental coordination disorder
}

\author{
Clarice Ribeiro Soares Araújo무 Lívia de Castro Magalhães ${ }^{2}$, \\ Ana Amélia Cardoso ${ }^{3}$
}

\begin{abstract}
ARAUJO, C. R. S.; MAGALHÃES, L. C.; CARDOSO, A. A. Uso da cognitive orientation to daily occupational performance (co-op) com crianças com transtorno do desenvolvimento da coordenação. Rev. Ter. Ocup. Univ. São Paulo, v. 22, n. 3, p. 245-253, set./dez. 2011.
\end{abstract}

\begin{abstract}
RESUMO: Crianças com Transtorno do Desenvolvimento da Coordenação (TDC) têm problemas motores que dificultam a realização de atividades diárias. Muitas abordagens são usadas para tratar estas crianças. O objetivo deste estudo foi explorar o uso da Cognitive Orientation to Daily Occupational Performance ou CO-OP em crianças brasileiras com TDC. Participaram do estudo três crianças de 9 a 10 anos com problemas de coordenação motora que interferiam no desempenho ocupacional em casa e na escola. Para avaliação das crianças foram utilizados o Questionário de Transtorno do Desenvolvimento da Coordenação (DCDQ-Brasil), o Movement Assessment Battery for Children (MABC), o Perceived Efficacy and Goal Setting System (PEGS), e a Medida Canadense de Desempenho Ocupacional (COPM), além da Análise Dinâmica do Desempenho (PQRS). O protocolo do CO-OP foi adaptado e a intervenção consistiu de 13 sessões de 60 minutos, duas vezes por semana, nas quais cada criança aprendeu três tarefas de sua escolha. Os resultados indicaram que o CO-OP pode ser uma abordagem eficaz, pois contribuiu para a melhora no desempenho funcional das crianças em cinco das seis tarefas escolhidas.
\end{abstract}

DESCRITORES: Transtornos das habilidades motoras; Estudos de intervenção; Avaliação de desempenho/métodos; Terapia ocupacional; Questionários/utilização.

\footnotetext{
* Projeto referente ao trabalho de conclusão de curso de especialização em Terapia Ocupacional com ênfase em Desenvolvimento Infantil da primeira autora. Trabalho apresentado na XXXVIII Reunião Anual da Sociedade Brasileira de Psicologia, 2008, Uberlândia-MG e no Congresso Brasileiro de Ensino e pesquisa em Saúde da criança e do adolescente, 2008, Rio de Janeiro, RJ.

1.Terapeuta ocupacional, especialista em Desenvolvimento Infantil e mestre em Ciências da Reabilitação.

2.Terapeuta ocupacional, professora titular do Departamento de Terapia Ocupacional da UFMG, PhD.

3.Terapeuta ocupacional, professora assistente do Departamento de Terapia Ocupacional da UFPR, doutoranda em Ciências da Reabilitação.

Endereço para correspondência: Rua Alvarenga, nº 384, bairro Cabeças, CEP 35400-000, Ouro Preto-MG. E-mail: clarice_risoar@, yahoo.com.br.
} 


\section{INTRODUÇÃO}

$\mathrm{U}$ ma queixa bastante comum em escolas infantis e consultórios de pediatria é a dificuldade apresentada por algumas crianças na realização de atividades que requerem habilidades motoras (HAMILTON, 2002). Estas crianças se movimentam de maneira incoordenada e parecem não saber como usar seus movimentos em atividades e brincadeiras em casa e na escola (MISSIUNA et al., 2004a). Geralmente essas crianças são lentas para escrever e o produto final é ilegível e desorganizado (MISSIUNA et al., 2004a). Elas precisam de mais tempo para ficar prontas para o intervalo escolar e, quando estão no parque ou na educação física, são os últimos a serem escolhidos pelos pares para participar de jogos e atividades motoras (MISSIUNA et al., 2004a).

Muitas vezes, essas crianças são descritas como "desajeitadas" ou "estabanadas", pois têm dificuldade para aprender e desempenhar tarefas rotineiras, como vestir roupa, escrever, andar de bicicleta ou jogar bola (CERMAK; LARKIN, 2002; HAMILTON, 2002). Muitos termos já foram usados para descrever este problema, tais como "síndrome da criança desajeitada", "apraxia e agnosia do desenvolvimento", "disfunção cerebral mínima", "paralisia cerebral mínima", "dispraxia do desenvolvimento". Atualmente o termo "Transtorno do Desenvolvimento da Coordenação (TDC)", encontrado no DSM IV sob o código 315.4 (APA, 2002), é o mais usado para se referir aos problemas de coordenação motora na criança (MAGALHÃES et al., 2006).

Estudos epidemiológicos estimam que a prevalência do TDC é de 6,4\%, afetando mais meninos do que meninas, não importando o status socioeconômico ou educacional (APA, 2002). O diagnóstico do transtorno geralmente é feito entre as idades de 6 e 12 anos e os critérios para diagnóstico são: (a) desempenho motor substancialmente abaixo do esperado para a idade cronológica e inteligência da criança; (b) o prejuízo motor interfere significativamente no rendimento escolar e no desempenho das atividades de vida diária; (c) o transtorno não é devido a uma condição médica geral (ex.: paralisia cerebral, hemiplegia, distrofia muscular), nem satisfaz critérios para transtorno invasivo do desenvolvimento; e (d) na presença de retardo mental, as dificuldades motoras são mais graves do que o esperado.

Alguns autores descrevem as crianças com TDC como uma população de características heterogêneas, distribuídas ao longo de um continuum de habilidades funcionais (NIEMEIJER et al., 2007). Parte dessa heterogeneidade se deve ao fato de que é comum a coocorrência com outros transtornos, como o transtorno de déficit de atenção/hiperatividade (TDA/H), dificuldades de leitura e problemas de linguagem (NIEMEIJER et al., 2007; DEWEY et al., 2002).

Apesar de existirem algumas teorias sobre a etiologia do TDC, ainda não há respostas definitivas sobre suas causas (SKINNER; PIEK, 2001). Investigações indicam que não há patologia ou alteração neurológica grosseira, pois não são encontradas anomalias morfológicas macroscópicas. Entretanto, funções anormais de transmissores e receptores no sistema nervoso central e o papel do cerebelo na disfunção de adaptação motora, são tópicos de algumas pesquisas (SKINNER; PIEK, 2001; LOOSE et al., 1991).

Os dados de vários estudos de seguimento com coortes de crianças com história de prematuridade demonstram maior prevalência do TDC e mais problemas de aprendizagem do que o esperado para a população geral (SUGDEN, 2007; MANDICH et al., 2001a; WILSON, 2005). Há evidências de que muitas crianças não superam o TDC e, quando atingem a adolescência e idade adulta, além dos problemas motores, observa-se maior freqüência de isolamento social, depressão, baixa auto-estima, baixo senso de eficácia e ansiedade (MÄNNISTÖ, 2006; WARD; RODGER, 2004; CHRISTIANSEN, 2000; BOUFFARD; WALL, 1990).

Uma vez que o TDC tem impacto ao longo da vida das pessoas, dificultando o desempenho tanto em atividades funcionais em casa e na escola, como nas atividades de lazer e socialização, é importante criar recursos para manejo do problema. Segundo Sugden (2007) existem basicamente duas abordagens de tratamento do TDC. As abordagens de processo se baseiam na premissa de que os problemas motores são ocasionados por déficits na integridade do sistema nervoso central (GENTILE, 1992). Exemplos de abordagens de processo são a Terapia de Integração Sensorial, o Tratamento Orientado para o Processo, o Treino Percepto-Motor e combinações entre estas. Abordagens de produto, por sua vez, são mais recentes, se baseiam na ciência do movimento humano e enfatizam o uso de estratégias de resolução de problemas para aquisição de habilidades funcionais. Estudos utilizando abordagens como a Motora Cognitiva ou a Intervenção Específica para a Tarefa, apontam que estas abordagens são eficazes para melhorar o aprendizado de tarefas e o desempenho funcional em crianças com TDC (MANDICH et al., 2001a; WILSON, 2005; NIEMEIJER et al., 2007; MÄNNISTÖ et al., 2006; WARD; RODGER, 2004).

Uma abordagem motora cognitiva, apontada pela literatura como das mais promissoras no tratamento do TDC, é o programa CO-OP (Cognitive Orientation to Daily Occupational Performance), cuja sigla original será mantida 
na Língua Portuguesa para não perder o sentido original de cooperação criança-terapeuta, uma das bases deste modelo (WILSON, 2005; MANDICH et al., 2001a,b). O CO-OP foi criado por terapeutas ocupacionais canadenses, com base em teorias da psicologia comportamental e cognitiva e na ciência do movimento humano. O programa é centrado na criança e tem como objetivos: a aprendizagem de atividades de interesse da criança, o uso de estratégias cognitivas, generalização e transferência do aprendizado (MANDICH; POLATAJKO, 2004).

No CO-OP a criança aprende a realizar três tarefas de sua escolha por meio de um processo de resolução de problemas centrado em quatro estratégias globais - meta, plano, faz, checa (MANDICH; POLATJKO, 2004). O terapeuta participa como mediador e encoraja a criança a refletir sobre seu desempenho e corrigir planos que não funcionaram. A participação dos pais é essencial neste programa, pois com o suporte dos pais a criança pode transferir e generalizar o uso das estratégias globais para outras tarefas, em diferentes contextos (MANDICH; POLATAJKO, 2004; POLATAJKO; CANTIN, 2006).

Os estudos conduzidos até o momento com o COOP, reportaram melhora no desempenho funcional dos participantes, aprendizagem e aplicação das estratégias cognitivas, e alguma evidência de generalização e transferência do aprendizado e manutenção dos ganhos a médio prazo (WILCOX; POLATAJKO, 1993; MARTINI; POLATAJKO, 1998; MILLER et al., 2001; DAWSON et al., 2009). No Brasil esta abordagem ainda é pouco conhecida e não é usada por terapeutas ocupacionais. Deste modo, são objetivos deste estudo: (a) fazer aplicação experimental da terapia motora cognitiva, especificamente com o protocolo $\mathrm{CO}-\mathrm{OP}$, em crianças brasileiras com TDC; (b) verificar se existe diferença significativa no desempenho em tarefas relevantes antes e depois do programa de intervenção; e (c) verificar se é necessário fazer adaptações no protocolo original do programa para melhor aplicação em crianças brasileiras.

\section{MATERIAIS E MÉTODO}

Como este é o primeiro trabalho brasileiro de aplicação do CO-OP, optou-se por utilizar o estudo de caso. Trata-se de um desenho de pesquisa descritivo, que reporta informações detalhadas tanto dos participantes quanto da intervenção, podendo sinalizar boas estratégias para o tratamento de indivíduos ou grupos de pacientes com determinada condição de saúde (OTTENBACHER, 1986). Essa mesma metodologia foi usada no desenvolvimento do CO-OP (MANDICH; POLATAJKO, 2004; WILCOX,
POLATAJKO, 1993; MARTINI; POLATAJKO, 1998; MILLER et al., 2001).

\section{Participantes}

Para participar do estudo, crianças de 9 e 10 anos de idade, com queixa de dificuldade de coordenação motora foram recrutadas em consultórios e clínica-escola de terapia ocupacional através de encaminhamentos feitos por profissionais da área cientes da natureza do estudo. A partir disto, estas crianças foram avaliadas com base nos critérios do DSM-IV para inclusão no estudo: (a) escore indicativo de TDC nos testes motores Developmental Coordination Disorder Questionnaire - DCDQ (WILSON et al., 2000) e Movement Assessment Battery for Children (HENDERSON; SUGDEN, 1992), (b) sem sinais de déficit cognitivo, aqui definido como desempenho acadêmico escolar dentro do esperado para a idade, de acordo com relato da professora, (c) sem diagnóstico de quadros clínicos ou doenças neurológicas específicas feito pelo pediatra, como reportado pelos pais.

\section{Instrumentação}

Para identificação de crianças com TDC foram aplicados dois testes motores. O Developmental Coordination Disorder Questionnaire - DCDQ (WILSON et al., 2000) é um questionário de pais, de fácil aplicação, com apenas 15 itens, específico para triagem de TDC, que recentemente foi traduzido e adaptado para o português, resultando no Questionário do Transtorno da Coordenação - DCDQ-Brasil (PRADO et al., 2009). O DCDQ é pontuado com escala Likert, de 5 pontos, que vai de "muito pior do que crianças da mesma idade" (escore 1) até a descrição "igual ou melhor do que crianças da mesma idade" (escore 5). A pontuação máxima, somando a pontuação das quatro áreas, é de 75 , sendo que o ponto de corte para identificação de TDC é pontuação $\leq 55$ para a idade de 8 anos a 9 anos e 11 meses e para a idade de 10 anos a 15 anos e 6 meses pontuação $\leq$ que 57. Crianças com pontuação acima dos valores indicados são consideradas como apresentando desenvolvimento típico. $\mathrm{O}$ DCDQ-Brasil foi usado tanto para triagem de crianças para o estudo como para reportar as dificuldades observadas no desempenho das ocupações diárias.

O Movement Assessment Battery for Children $M A B C$ (HENDERSON; SUGDEN, 1992) é um dos testes mais usados em pesquisa para diagnóstico de TDC (GEUZE, 2003; WILSON, 2005). O MABC já foi validado em diferentes países e apresenta bons índices de confiabilidade teste-reteste $(0,75)$ e entre-examinadores $(0,70)$ (SKINNER; 
PIEK, 2001). É feita observação direta do desempenho da criança em oito tarefas nas áreas de destreza manual, habilidades com bola e equilíbrio dinâmico e estático. Quanto mais elevada a pontuação da criança, pior o seu desempenho no teste. O MABC foi usado para confirmar o diagnóstico, sugerido pelo DCDQ-Brasil, e para avaliar o desempenho motor das crianças antes e depois da intervenção. Só foram incluídas no estudo crianças que obtiveram escore total no MABC abaixo do $5^{\circ}$ percentil, que sinaliza presença definitiva de problemas motores.

Para definir as metas de tratamento foi usado o Perceived Efficacy and Goal Setting System - PEGS (MISSIUNA et al., 2004b), que é um procedimento de entrevista que usa figuras para ajudar a criança a identificar tarefas nas quais tenha dificuldade. O PEGS combina a perspectiva da criança com informações dadas pelos pais e professores. O instrumento tem boa validade e confiabilidade (MISSIUNA et al., 2004), já foi traduzido e adaptado para o português, e está disponível para pesquisa (BIGONHA, 2008). As metas selecionadas pelas crianças foram pontuadas com o sistema de pontuação da Canadian Occupational Performance Measure - COPM (LAW et al., 2009), em escala de 1 a 10, para graduar a qualidade do desempenho nas metas e o nível de satisfação, antes e depois da intervenção. $\mathrm{O}$ nível de desempenho nas metas também foi avaliado por meio de filmagem, sendo pontuado por um avaliador externo com uso da Performance Quality Rating Scale PQRS (POLATAJKO; MANDICH, 2004), uma escala de 1 a 10 , criada especificamente para uso no programa CO-OP. Esta escala avalia, também, a magnitude da mudança no desempenho ( 5 vezes pior a 5 vezes melhor).

\section{Procedimentos}

Inicialmente foi realizado estudo piloto com uma menina de 9 anos, de acordo com o protocolo de intervenção do CO-OP (POLATAJKO; MANDICH, 2004): a sessão 1 destinada à administração do PEGS, da COPM e à filmagem da criança, seguida de pontuação da $\mathrm{PQRS}$ pelas próprias pesquisadoras; na sessão 2 , a terapeuta responsável pela condução da intervenção introduziu as estratégias globais META-PLANO-FAZ-CHECA; nas sessões de 3 a 11 foi realizada a intervenção; na sessão 12 , a criança foi filmada novamente fazendo as atividades aprendidas e a PQRS foi preenchida. Com base neste estudo piloto, foi verificada a necessidade de se providenciar mais material informativo para os pais, bem como o acréscimo de uma sessão na metade do programa de tratamento, para tirar dúvidas, rever procedimentos a reforçar orientações aos pais. A partir de então, a pesquisa foi conduzida como descrito na Tabela 1.

Tabela 1 - Estrutura das sessões do CO-OP

\begin{tabular}{l|l}
\hline Antes da terapia & $\begin{array}{l}\text { Contato com os pais para verificar o interesse em participar do estudo, checar os dados clínicos e } \\
\text { excluir crianças com sinais neurológicos ou diagnóstico específico dado pelo pediatra. } \\
\text { Triagem inicial dos candidatos com uso do DCDQ-Brasil. SNAP IV respondido pelas professoras. } \\
\text { Avaliação motora com aplicação do MABC. }\end{array}$ \\
\hline Sessão 1 & $\begin{array}{l}\text { Definição de metas e avaliação do desempenho da criança: aplicação do PEGS, COPM e PQRS. } \\
\text { Filmagem da criança realizando as três tarefas escolhidas. }\end{array}$ \\
\hline Sessão 2 & Introdução do fantoche Mr. MetaPlanoFazCheca, que explica e demonstra as estratégias globais \\
\hline Sessão 3 a 6 e 8 a 12 & $\begin{array}{l}\text { Aquisição de habilidades: uso das estratégias globais e técnicas de mediação, com descoberta guiada } \\
\text { de estratégias específicas para melhorar o desempenho nas tarefas. Neste momento, a criança é } \\
\text { levada a pensar sobre a tarefa e maneiras de fazê-la, sendo direcionada pela terapeuta na descoberta } \\
\text { de estratégias que podem facilitar o desempenho. Educação de pais. }\end{array}$ \\
\hline Sessão 7 & Discussão com os pais sobre a generalização e transferência do uso das estratégias em casa. \\
\hline Sessão 13 & $\begin{array}{l}\text { Avaliação final da criança: aplicação da COPM e PQRS. } \\
\text { Filmagem da criança realizando as tarefas. }\end{array}$ \\
\hline Após a intervenção & Administração do MABC. \\
\hline
\end{tabular}

O MABC foi aplicado por examinador externo treinado nos procedimentos de avaliação e que não conhecia os objetivos de terapia selecionados por cada criança. A testagem foi filmada para permitir checagem da correção dos escores. A pontuação da PQRS também foi feita por examinador externo, com base em filmagem do desempenho 
em cada atividade definida como meta, sendo que os segmentos das duas crianças foram misturados, para não permitir identificar se era antes ou depois da intervenção. As sessões tiveram duração de 60 minutos, duas vezes por semana e aconteceram nas dependências da Escola de Educação Física, Fisioterapia e Terapia Ocupacional da UFMG em locais escolhidos de acordo com as tarefas. Os pais foram convidados a assistir às sessões.

Durante a segunda sessão as estratégias globais META, PLANO, FAZ, CHECA foram ensinadas e um fantoche foi apresentado para as crianças. $\mathrm{O}$ fantoche $-\mathrm{Mr}$. MetaplanoFazcheca - é uma estratégia mnemônica para ajudar a criança a usar as estratégias globais para descobrir soluções para os problemas que enfrenta na realização das tarefas. O fantoche é apresentado como um detetive que fareja soluções para os problemas e, assim que a criança compreende o significado de cada palavra (meta: o que eu quero fazer; plano: como vou fazer; faz: execução do plano; checa: verificar se o plano funcionou ou não), terapeuta e criança criam exemplos de como usar estas estratégias em alguma atividade conhecida. Uma vez aprendidas, as estratégias globais serão aplicadas às diferentes metas, sendo então descobertas estratégias específicas (como posicionamento do corpo na realização da tarefa, adaptações da tarefa, atenção ao fazer, estratégias de memória, entre outras - ex.: "para atirar e acertar a bola no gol, eu tenho que "grudar" os olhos no alvo") ao longo das outras sessões. Todas as sessões foram filmadas, uma vez que o desempenho ocupacional das crianças era constantemente analisado de forma dinâmica, assim como previsto no protocolo de intervenção original (POLATAJKO; MANDICH, 2004). As famílias foram informadas do caráter experimental do estudo, cujo projeto foi aprovado pelo Comitê de Ética em Pesquisa da Universidade Federal de Minas Gerais (Parecer $n^{\circ}$ ETIC 333/07). Os pais e as crianças assinaram termo de consentimento livre e esclarecido e autorizaram a execução de fotos e filmagem, antes de participarem da pesquisa.

\section{RESULTADOS}

Participaram deste estudo três crianças, uma menina de 9 anos de idade e dois meninos de 9 e 10 anos de idade. Serão descritos apenas os resultados dos dois meninos que participaram do estudo principal, uma vez que a menina fez parte do estudo piloto. As metas dos meninos estão descritas na Tabela 2.

Tabela 2 - Metas dos participantes

\begin{tabular}{lllll}
\hline Criança & Idade & Meta 1 & Meta 2 & Meta 3 \\
\hline A & 9 anos & Agarrar bola & Corrida & Escrita \\
$\mathrm{R}^{*}$ & 10 anos & Agarrar bola & Fazer cestas & Pintura \\
\hline
\end{tabular}

*Criança com Transtorno do Déficit de Atenção/Hiperatividade diagnosticado pelo neurologista infantil.

Os escores e a diferença entre as avaliações realizadas antes e depois da intervenção são apresentados na Tabela 3.

Tabela 3 - Resultados da análise de desempenho, satisfação e do teste MABC antes e depois da intervenção

\begin{tabular}{|c|c|c|c|c|c|c|c|}
\hline \multirow[b]{2}{*}{ Medidas } & \multicolumn{3}{|c|}{ Criança A } & \multirow[b]{2}{*}{ Medidas } & \multicolumn{3}{|c|}{ Criança R } \\
\hline & Antes & Depois & Diferença & & Antes & Depois & Diferença \\
\hline PQRS agarrar bola & 2 & 7 & +5 & PQRS agarrar bola & 4 & 7 & +3 \\
\hline PQRS correr & 3 & 6 & +3 & PQRS fazer cestas & 2 & 2 & 0 \\
\hline PQRS escrita & 6 & 8 & +2 & PQRS pintar & 5 & 8 & +3 \\
\hline COPM(D) agarrar bola & 4 & 8 & +4 & COPM(D) agarrar bola & 3 & 10 & +7 \\
\hline COPM(S) agarrar bola & 5 & 9 & +4 & COPM(S) agarrar bola & 5 & 10 & +5 \\
\hline COPM(D) correr & 5 & 7 & +2 & COPM(D) fazer cestas & 3 & 9 & +6 \\
\hline COPM(S) correr & 6 & 8 & +2 & COPM(S) fazer cestas & 5 & 10 & +5 \\
\hline COPM(D) escrita & 3 & 7 & +4 & COPM(D) pintar & 1 & 10 & +9 \\
\hline COPM(S) escrita & 5 & 6 & +1 & COPM(S) pintar & 1 & 9 & +8 \\
\hline MABC habilidades com bola & 3 & 1 & -2 & MABC habilidades com bola & 8 & 5 & -3 \\
\hline MABC destreza manual & 7.5 & 9.5 & +2 & MABC destreza manual & 12 & 11 & -1 \\
\hline MABC equilíbrio & 3.5 & 1 & $-2,5$ & MABC equilíbrio & 7 & 2.5 & $-5,5$ \\
\hline MABC total & 14 & 11.5 & $-2,5$ & MABC total & 27 & 18.5 & $-8,5$ \\
\hline
\end{tabular}

Nota: $\operatorname{COPM}(\mathrm{S})$ = nível de satisfação; $\operatorname{COPM}(\mathrm{D})$ = qualidade do desempenho. Pontuação positiva relativa à diferença na PQRS e nos itens da $\mathrm{COPM}$ indica melhora. No MABC, quanto mais alto o resultado, pior o desempenho motor, assim diferença negativa indica melhora, enquanto positiva indica desempenho pior no pós-teste. 


\section{DISCUSSÃO}

De acordo com a PQRS, a criança A obteve melhora no desempenho em todas as atividades, enquanto a criança $\mathrm{R}$ conseguiu melhorar o desempenho em duas das atividades escolhidas. A análise dos escores da COPM mostra que as crianças perceberam melhora e se mostraram mais satisfeitas com o próprio desempenho em todas as tarefas aprendidas.

Como diferenças de dois ou mais pontos na COPM são clinicamente importantes (LAW et al., 2009), na Tabela 3 podemos observar que houve diferença clinicamente significativa nas pontuações da COPM em todos os itens, exceto no escore de satisfação da criança A com o desempenho na escrita. Observa-se, ainda, que para a criança $\mathrm{A}$, os dados da PQRS e da COPM são consistentes entre si e apontam melhoria no desempenho. A criança $\mathrm{R}$ percebeu melhora no desempenho em todas as tarefas, no entanto, há incongruência com a avaliação (PQRS) do examinador externo na meta de fazer cestas, pois este não percebeu melhoria no desempenho.

Os resultados negativos do MABC indicam melhora nas habilidades motoras, com diminuição dos escores em quase todos os itens, com exceção daqueles que testam destreza manual da criança A. Apesar das diferenças encontradas antes e depois da intervenção, as crianças não obtiveram escore total suficiente para mudarem de percentil, indicando que o TDC persiste.

As mudanças nos escores de satisfação do COPM mostram que, apesar dos problemas motores persistirem após a intervenção, como apontado pelo $\mathrm{MABC}$, as crianças se mostraram mais satisfeitas em relação ao desempenho de atividades que realizam no ambiente escolar. Tal resultado era esperado, uma vez que na terapia não foram abordadas atividades específicas do MABC. O protocolo do CO-OP tem foco no desempenho funcional, partindo da premissa de que uma vez que a criança consegue realizar as atividades de seu interesse e cria estratégias para resolver seus problemas motores, torna-se mais fácil conviver com o TDC.

É importante ressaltar que as tarefas escolhidas foram atividades típicas da infância e, principalmente, do ambiente escolar. Habilidades como agarrar bola e fazer cestas fazem parte de esportes em grupo comumente ensinados nas escolas brasileiras, como o futebol, basquete e queimada.

Dificuldades nos esportes e outras atividades que demandam coordenação motora podem gerar sentimentos de frustração nas aulas de educação física e no intervalo escolar. Crianças com TDC participam menos de atividades físicas estruturadas como os esportes, por sentirem medo de serem ridicularizadas por seus colegas (CAIRNEY et al.,
2007). Muitas delas acham difícil conviver com os colegas, pois eles passam o recreio jogando futebol, pulando corda ou engajados em outras atividades físicas típicas, que são difíceis para quem tem problemas de coordenação e, por isso, as crianças com TDC permanecem, frequentemente, sozinhas ou brincam com crianças mais jovens (MISSIUNA et al., 2007). Segundo Fitzpatrick e Watkinson (2003), sentimentos de insegurança, medo, raiva e humilhação são relatados por adultos que vivenciaram experiências desastrosas devido aos problemas de coordenação motora na infância.

Em relação à utilização das estratégias globais e aprendizagem das estratégias específicas, pode-se observar sinais de generalização para outros ambientes e tarefas, de acordo com relatos das mães. A mãe da criança A reportou que "Ele se entusiasmou com as estratégias para resolver questões comuns da infância. Nossa família percebe o tanto que ele está feliz. A não queria faltar às sessões e fala das estratégias com sua professora". Além disto, a mãe de A. relatou melhoras na escola, particularmente na cópia de trabalhos escritos, de acordo com observações feitas pela professora.

A mãe da criança $\mathrm{R}$ reportou que " $R$ precisava de direcionamento para aprender e com esta abordagem é possível fazer em casa. A nossa participação foi positiva e muito válida. Mas, percebi poucas melhoras em casa, pois ele ainda está resistente para usar as estratégias comigo."

É importante ressaltar que o fato de $\mathrm{R}$ ter também TDA/H pode ter contribuído para que se mantivesse por menos tempo focado nas tarefas e na aprendizagem das estratégias, contribuindo para que ele tenha maior dificuldade na transferência e generalização das estratégias globais e específicas para outras atividades em casa e na escola.

Os resultados obtidos são consistentes com trabalhos descritos na literatura, que indicam que o uso de estratégias cognitivas pode ser um recurso muito útil para o tratamento de crianças com TDC (MARTINI; POLATAJKO, 1998; WARD; RODGER, 2004; MANDICH; POLATAJKO, 2004). Os resultados positivos na maioria das avaliações no pós-teste dão suporte ao fato do $\mathrm{CO}-\mathrm{OP}$ ser considerado um dos protocolos mais promissores para o manejo dos problemas enfrentados por estas crianças (MANDICH et al., 2001b; WILSON, 2005; POLATAJKO; CANTIN, 2006; SUGDEN, 2007).

No Brasil, esta foi a primeira tentativa de replicação de estudos estrangeiros, sendo que o estudo piloto mostrou a necessidade de serem feitas algumas adaptações no protocolo original. Uma vez que existe pouca literatura em português sobre programas motores cognitivos, foi 
considerado importante acrescentar uma sessão de orientação para os pais, na qual foram esclarecidas dúvidas sobre o TDC e sobre o uso de estratégias cognitivas, na tentativa de estimular a continuidade ao programa em casa. Essa modificação foi positiva, pois os pais se empenharam no uso das estratégias em casa, o que não ocorreu no estudo piloto. É possível que ainda sejam necessárias mais adaptações direcionadas ao treinamento de pais e cuidadores, o que deve ser investigado em estudos futuros.

O uso da metodologia de estudo de caso são recomendados na aplicação e avaliação de novas abordagens clínicas aplicadas (OTTENBACHER, 1986). O fato deste ser um estudo exploratório com apenas duas crianças limita a generalização dos resultados. Por isto, recomenda-se mais investigações sobre o uso do CO-OP incluindo número maior de crianças. Além disso, pesquisas futuras poderiam analisar a eficácia desta abordagem no tratamento de crianças com TDC associado ao TDA/H, investigar o tempo de permanência das crianças na realização de tarefas, bem como a freqüência com que utilizam as estratégias globais e específicas na resolução de problemas de desempenho. Outro aspecto importante seria acompanhar as crianças tratadas, ao longo do tempo, para verificar se elas persistem usando as estratégias aprendidas.

\section{CONCLUSÃO}

Estudos que melhorem a compreensão dos problemas enfrentados por crianças com TDC e investigar alternativas efetivas de tratamento são muito importantes, uma vez que os encaminhamentos desta clientela para terapeutas ocupacionais e fisioterapeutas vêm aumentando (MISSIUNA et al., 2006). As evidências apontam para eficácia das abordagens orientadas para o produto, nas quais se inserem as abordagens cognitivas.

No Brasil, torna-se ainda mais importante a investigação deste tipo de abordagem, pois se trata de intervenção breve - tempo de execução está em torno de 04 meses - que avalia consistentemente os resultados obtidos, em congruência com a prática baseada em evidências. Além disto, percebe-se que são necessários poucos recursos para aplicá-la - as ferramentas de terapia são materiais comumente utilizados pelas crianças no cotidiano.

Este estudo é o primeiro passo para a adequação do uso do CO-OP no tratamento de crianças com TDC no nosso país. Nesta primeira experiência algumas modificações foram feitas para melhor adaptação à nossa realidade, como o acréscimo de uma sessão ao protocolo destinada ao fornecimento de mais informações sobre a terapia e o TDC. Os dois casos documentados demonstraram que as crianças conseguiram melhorar o desempenho em tarefas diárias significativas para elas. Espera-se que os resultados obtidos incentivem o uso desta terapia no ambiente clínico, a investigação com amostras mais amplas e análise dos efeitos desta terapia em longo prazo.

Agradecimentos: a todos envolvidos neste trabalho, em particular às crianças e suas famílias que aceitaram participar, além das terapeutas ocupacionais e estagiárias que contribuíram com boa vontade e seus conhecimentos.

ARAujo, C. R. S.; MAGAlhãeS, L. C.; CARDOSO, A. A. Cognitive orientation to daily occupational performance (co-op) with children with developmental coordination disorder. Rev. Ter. Ocup. Univ. São Paulo, v. 22, n. 3, p. 245-253, set./dez. 2011.

\begin{abstract}
Children with Developmental Coordination Disorder (DCD) have motor problems that lead to difficulties to perform every day tasks. Many approaches are used to treat these children. The aim of this study is to explore the use of the Cognitive Orientation to Daily Occupational Performance (CO-OP) with Brazilian children with DCD. Three children ages 9 to 10 years old with motor coordination problems that interfered in occupational performance at home and school participated in the study. They were assessed using the Developmental Coordination Disorder Questionnaire-Brazil (DCDQ-Brazil), the Movement Assessment Battery for Children (MABC), the Perceived Efficacy and Goal Setting System (PEGS) and the Canadian Occupational Performance Measure (COPM); the Performance Quality Rating Scale (PQRS) was also used. The CO-OP's protocol was adapted. The intervention lasted 13 sessions, twice a week, and the children learned three tasks of their choice. The results indicate that CO-OP can be an effective approach, because it contributed to improvement in occupational performance on five of six of the chosen tasks.
\end{abstract}

KEY WORDS: Motor skills disorders; Intervention studies; Employee performance appraisal/ methods; Occupational therapy; Questionnaires/utilization. 


\section{REFERÊNCIAS}

AMERICAN PSYCHIATRIC ASSOCIATION. Manual diagnóstico e estatístico de transtornos mentais: DSM-IV-TR. Trad. Jorge, M.R. 4a. ed. Porto Alegre, RS: Artes Médicas, 2002.

BIGONHA, C. I. Adaptação transcultural do Perceived Eficcacy and Goal Setting Scale (PEGS) para crianças de 6 a 9 anos de idade. Belo Horizonte, 2008. [Dissertação] Programa de Mestrado em Ciências da Reabilitação, UFMG.

BOUFFARD, M.; WALL, A. E. A problem solving approach to movement skill acquisition: implications for special populations. In: REID, G. Problems in movement control. North-Holland: Elsevier Science, 1990.

CAIRNEY, J.; HAY, J.; MANDIGO, J.; WADE, T.; FLOURIS, A. Developmental coordination disorder and reported enjoyment of physical education in children. Eur. Phys. Educ. Rev., v. 13, n. 1, p. 81-98, 2007.

CERMAK, S. A.; LARKIN, D. Developmental coordination disorder. Albany, NY: Delmar Thomson Learning, 2002.

CHRISTIANSEN, A. S. Persisting motor control problems in 11 to 12 year old boys previously diagnosed with déficits in attention, motor control and perception. Dev. Med Child Neurol., v. 42, p. 4-7, 2000.

DAWSON, D. R.; GAYA, A.; HUNT, A.; LEVINE, B.; LEMSKY, C.; POLATAJKO, H. J. Using the cognitive orientation to occupational performance (CO-OP) with adults with executive dysfunction following traumatic brain injury. Can. JOccup. Ther, v. 76, n. 2 , p. 115-27, 2009.

DEWEY, D.; KAPLAN, B. J.; CRAWFORD, S. G.; WILSON, B. N. Developmental coordination disorder: associated problems in attention, learning, and psychosocial adjustment. Human Mov. Sci., v. 21, p. 905-918, 2002.

FITZPATRICK, D. A.; WATKINSON, E. J. The lived experience of physical awkwardness: adults' retrospective views. Adapted Phys. Activity Q., v. 20, p. 279-297, 2003.

GENTILE, A. M. The nature of skill acquisition: therapeutic implications for children with movement disorders. Med. Sci. Sports Exercise, v. 36, p. 31-40, 1992.

GEUZE, R. H. Static balance and developmental coordination disorder. Human Mov. Sci., v. 22, n. 4-5, p. 527-548, 2003.

HAMILTON, S. S. Evaluation of clumsiness in children. Am. Family Phys., v. 66, n. 8, p. 1435-1440, 2002.

HENDERSON, S. E.; SUGDEN, D. The Movement Assessment
Battery for Children. Kent, UK: Psychological Corporation, 1992.

LAW, M.; et al. Medida Canadense de Desempenho Ocupacional (COPM). Org. Trad. Lívia de Castro Magalhães, Lílian Vieira Magalhães, Ana Amélia Cardoso. Belo Horizonte: Editora UFMG, 2009.

LOOSE, A.; HENDERSON, S. E.; ELLIMAN, D.; HALL, D.; KNIGTH, E.; JONGMANS, M. Clumssiness in children - do they grow out of it? A 10 year follow-up study. Dev. Med. Child Neurol., v. 33, p. 55-68, 1991.

MAGALHÃES, L. C.; MISSIUNA, C.; WONG, S. Terminology used in research reports of developmental coordination disorder. Dev. Med. Child Neurol., v. 48, n. 11, p. 937-41, 2006.

MANDICH, A. D.; POLATAJKO, H. J. Enabling occupation in children: the Cognitive Orientation to Daily Occupational Performance (CO-OP) Approach. Ottawa, ON: CAOT, 2004.

MANDICH, A. D.; POLATAJKO, H. J.; MACNAB, J. J.; MILLER, L. T. Treatment of children with developmental coordination disorder - what is the evidence? Phys. Occup. Ther. Pediatr., v. 20, n. 2-3, p. 51-68, 2001 a.

MANDICH, A. D.; POLATAJKO, H. J.; MISSIUNA, C.; MILLER, L. T. Cognitive strategies and motor performance in children with developmental coordination disorder. Phys. Occup. Ther. Pediatr., v. 20, n. 2-3, p. 125-143, 2001 b.

MÄNNISTÖ, J. P.; CANTELL, M.; HOUVINEN, T.; KOOISTRA, L.; LARKIN, D. A school-based intervention movement programme for children with motor learning difficulty. Eur. Phys. Educ. Rev., v. 12, n. 3, p. 273-287, 2006.

MARTINI, R.; POLATAJKO, H. J. Verbal self-guidance as a treatment approach for children with developmental coordination disorder: a systematic replication study. Occup. Ther. J. Res., v. 18, n. 4, 1998.

MILLER, L. T.; POLATAJKO, H. J.; MISSIUNA, C.; MANDICH, A. D.; MACNAB, J. J. A pilot trial of a cognitive treatment for children with developmental coordination disorder. Human Mov. Sci., v. 20, p. 183-210, 2001.

MISSIUNA, C.; RIVARD, L.; POLLOCK, N. They're bright but can't write: developmental coordination disorder in school aged children. Teach. Except. Child Plus, v. 1, n.1, 2004a.

MISSIUNA, C.; MOLL, S.; KING, S.; KING, G.; LAW, M. A trajectory of troubles: parents' impressions of the impact of developmental coordination disorder. Phys. Occup. Ther. Pediatr., v. 27, n. 1, p. 81-101, 2007. 
MISSIUNA, C.; MOLL, S.; LAW, M.; KING, S.; KING, G. Misteries and mazes: parents' experiences of children with developmental coordination disorder. Can. J. Occup. Ther., v. 73, n. 1, p. 7-17, 2006.

MISSIUNA, C.; POLLOCK, N.; LAW, M. Perceived Efficacy and Goal Setting System (PEGS). San Antonio, TX: Psychological Corporation, 2004b.

NIEMEIJER, A. S.; SMITS-ENGELSMAN, B. C. M.; SCHOEMAKER, N. M. Neuromotor task training for children with developmental coordination disorder: a controlled trial. Dev. Med. Child Neurol., v. 49, p. 406-411, 2007.

OTTENBACHER, K. J. Evaluating clinical change strategies for occupational and physical therapists. Baltimore: Williams \& Wilkins, 1986.

POLATAJKO, H. J.; CANTIN, N. Developmental Coordination Disorder (Dyspraxia): an overview of the state of the art. Semin. Pediatr. Neurol., v. 12, p. 250-258, 2006.

PRADO, M. S. S.; MAGALHÃES, L. C.; WILSON, B. N. Cross cultural adaptation of the Developmental Coordination Questionnaire for brazilian children. Rev. Bras. Fisioter, v. 13, n. 3, p. 236-46, 2009.

SKINNER, R. A.; PIEK, J. P. Psychossocial implications of poor motor coordination in children and adolescents. Human Mov. Sci., v. 20 , p. $73-94,2001$.

SUGDEN, D. Current approaches to intervention in children with developmental coordination disorder. Dev. Med. Child Neurol., v. 49, p. 467-471, 2007.

WARD, A.; RODGER, S. The application of Cognitive Orientation to daily Occupational Performance (CO-OP) with children 5-7 years with developmental coordination disorder. Br. J. Occup. Ther, v. 67, n. 6, 2004.

WILCOX, A. L.; POLATAJKO, H. J. Verbal self-guidance as a treatment technique for children with developmental coordination disorder. Can. J. Occup. Ther., v. 60, n. 20, 1993.

WILSON, B. N.; KAPLAN, B. J.; CRAWFORD, S. G.; CAMPBELL, A.; DEWEY, D. Reliability and validity of a parent questionnaire on childhood motor skills. Am. J. Occup. Ther, v. 54, p. 484-493, 2000.

WILSON, P. H. Practioner Review: Approaches to assessment and treatment of children with DCD: an evaluate review. J. Child Psychol. Psychiatr., v. 46, n. 8, p. 806-823, 2005. 